\title{
Only Binary Stars Can Help Us Actually SEE a Stellar Chromosphere
}

\author{
R. Elizabeth Griffin \\ Herzberg Institute for Astrophysics, DAO, Canada
}

\begin{abstract}
.
Binary stars of the $\zeta$ Aurigae type (eclipsing systems containing a cool giant plus a hot mainsequence star) offer a unique and highly effective method of probing a stellar chromosphere. Close to occultation the main-sequence star acts as a light probe behind the giant's chromosphere, enabling an observer to detect changing conditions in that chromosphere along the line of sight. The technique is powerful, the effects dramatic. However, presently known eclipsing systems number only about 10, and a much greater sample is required for meaningful statistics of the properties of stellar chromospheres. New surveys of fainter binaries should be investigated for eclipses in order to gain more information on chromospheres in general. Such information is vital for modelling stellar photospheres, from which abundances are derived. This paper describes the very different behaviour of chromospheric material in three $3^{\text {rd }}$-magnitude giants.
\end{abstract}

\section{Introduction}

A $\zeta$ Aur binary offers a unique opportunity to investigate the chromosphere of a cool giant directly, through observation and analysis of the phenomenon of "atmospheric eclipse". The power of the opportunity was realized over 70 years ago when observations of the first such eclipse, in $\zeta$ Aur itself, were published, but there has been only limited progress in modelling chromospheric spectra, despite the wealth which they promise. One downside of this research is the relatively small sample size (about 10) of known eclipsing systems. Worse, those systems which we have been able to investigate to date show very considerably variety, and - worse still! - events seen during one ingress or egress do not necessarily repeat at the next. Thus, instead of simply swelling the bank of information by pooling the results, we face the need to monitor each event over and over again in order to find out just what are the different and variable processes and structures whose signatures we are now slowly uncovering.

The need to understand a stellar chromosphere is becoming more pressing as our ability to model photospheres improves. Even today, if a contribution from the chromosphere is included at all (and usually it is just ignored!), the most that is done is to add a global parameter to allow for variation in the temperature gradient of the outer atmosphere.

\section{All Chromospheres are Different!}

Before the influence of a chromosphere can be introduced appropriately into a photospheric model, efforts have to be made to observe, analyse and try to understand a stellar chromosphere as a separate entity. It is precisely here that binary stars are indispensable. But we have a long way to go. Progress is slow, because eclipse events have to be monitored repeatedly and all but one of the 10 known $\zeta$ Aur binaries have a period well over a year; for 31 Cyg it is over 10 years.

The chromosphere has traditionally been treated as a homogeneous, spherical region that obeys a simple barometric law. In the low chromosphere such a law may be a 
reasonable approximation owing to the overall density there (lack of "empty space"). However, recent series of chromospheric spectra, observed when the secondary was a good fraction of the giant's radius outside eclipse ingress/egress, show clearly that the outer region of a giant or supergiant chromosphere is anything but homogeneous, and that it changes on time-scales of both days and years. The sequences of chromospheric features in $\zeta$ Aur, $31 \mathrm{Cyg}$ and $32 \mathrm{Cyg}$ selected for this poster display considerable differences in detail, despite the supposed similarity of the supergiants in question.

If stellar winds largely originate in those outer atmospheric regions, then here is another urgent need to understand the mechanism(s) causing the short-term changes seen in stellar chromospheres.

\section{Atmospheric Eclipses}

In order to measure and analyse the chromosphere of the luminous cool giant in a $\zeta$ Aur binary in detail through the phenomenon of atmospheric eclipse, the secondary spectrum must first be isolated from that of the composite spectrum, either by subtracting the spectrum of the giant (observed during totality) or by spectrum disentangling. What remains is the spectrum of the secondary star, with phase-dependent (i.e. heightdependent) chromospheric absorption features superimposed; Figures 1-3 show examples of the Ca II K-line region in different binaries. It then requires knowledge of the geometry of the binary in order to relate the chromospheric information to a particular location in the atmosphere of the cool giant in question. Although spectroscopy of stellar chromospheres has been pursued in the "classical" $\zeta$ Aur binaries ( $\zeta$ Aur, 31 Cyg and 32 Cyg) over the past 80 years, it was only after recent applications of digital techniques that real progress could be made towards isolating and analysing quantitatively the information contained in the chromospheric features.

Observational work has traditionally been limited to phases showing the low chromosphere, i.e., close to occultation, because that is where the absorption lines are strongest and most plentiful. The low chromosphere is relatively dense - it is in effect an extension of the photosphere. However, results from high-dispersion spectroscopy of even the lower chromospheres of several $\zeta$ Aur systems have demonstrated the existence of a twocomponent structure there, characterised by considerably disparate scale heights, as in a "core and halo".

In fact, fascinating information can also be gleaned by studying the chromosphere at much greater heights. At those phases the drama of the inner atmospheric eclipse has passed (or, for ingress, has not yet commenced), and the only chromospheric signatures that can be detected in ground-based spectra are in the cores of the very strongest photospheric lines, namely the Ca II H \& K lines. None of the historic sequences of atmospheric-eclipse spectra in astronomical archives has continued far enough, but we are now trying to observe each eclipse ingress and egress for several weeks, not just for a few days before and after totality.

In 1990 a 14-night egress series of spectra of $\zeta$ Aur $(\mathrm{K} 4 \mathrm{II}+\mathrm{B} 6.5 \mathrm{~V})$ led to the discovery that the Ca II $\mathrm{K}$ line and all other ionic lines bifurcated at a chromospheric height of $\sim 0.3$ of the primary's radius. But the splitting did not occur in all subsequent eclipses, thus eliminating a geometrical cause, though in 2003 the pattern almost repeated that seen in 1990 (Figures 1-3). Such a chromosphere may therefore be composed of relatively small-scale chromospheric structures, with a characteristic variability which is less than the temporal and spatial resolution afforded by the geometry of the binary. How can such features be modelled? Are we seeing separate clouds of absorbing material, or is the splitting actually the result of superimposed chromospheric emission? 

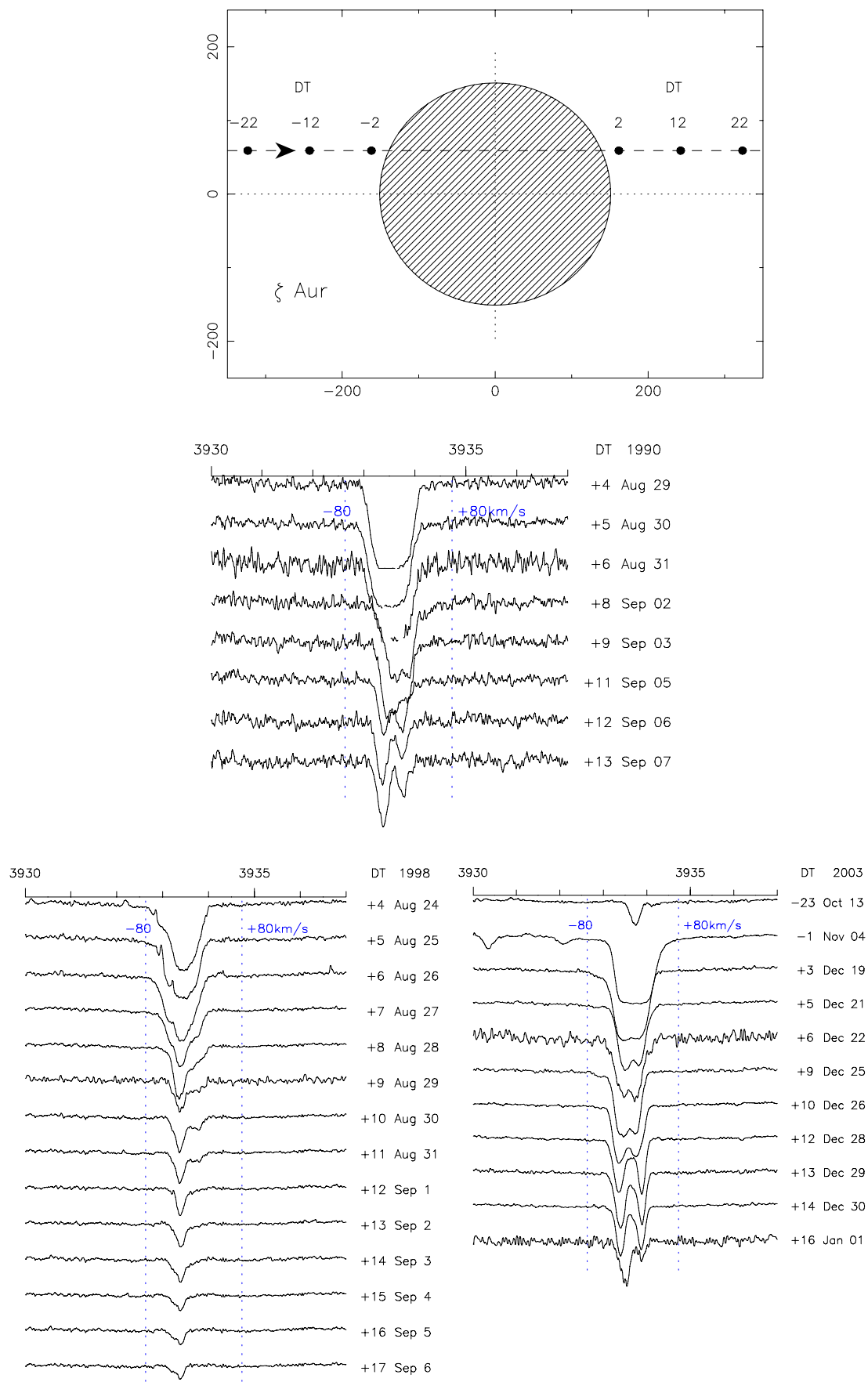

Figure 1. Top: Scaled model of $\zeta$ Aur, in units of $R_{\odot}$, near totality. The small dots represent the positions of the hot star at selected $D T$ relative to the giant; $D T$ is measured in days before $2^{\text {nd }}$ or after $4^{\text {th }}$ contact. The middle and lower pairs of spectra show the Ca II K line in $\zeta$ Aur B during chromospheric egress in 1990, 1998 and 2003, respectively, with dates and calculated DT. The dotted blue lines indicate velocity shifts of -80 and $+80 \mathrm{~km} / \mathrm{s}$ from the rest wavelength of the $\mathrm{K}$ line. 

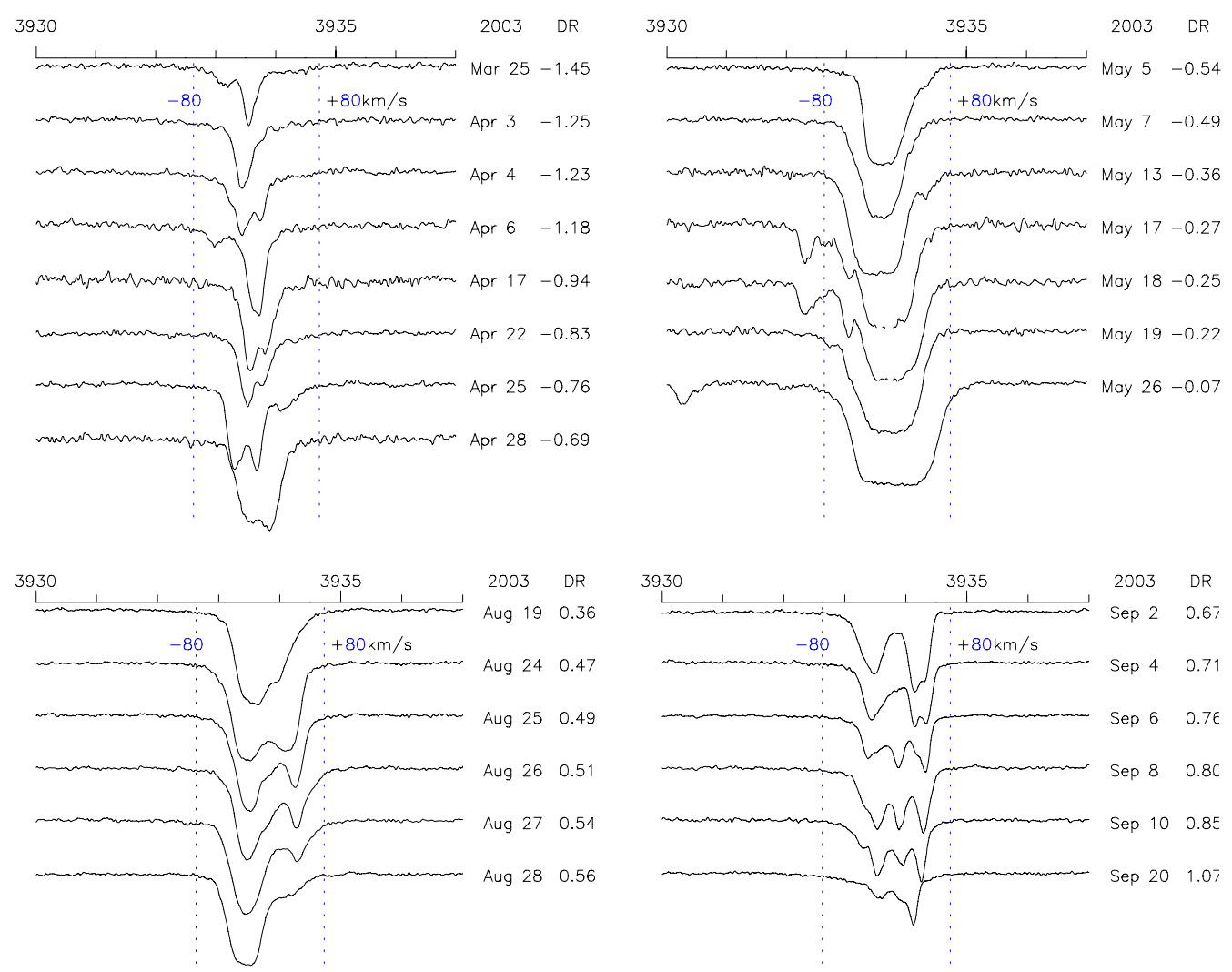

Figure 2. Changes in the chromospheric K line in 31 Cyg during ingress (upper plot) and egress (lower plot), observed at phases near totality in 2003. The quantity $D R$ after the date for each plot represents the height of the hot secondary from the limb of the primary, in units of the radius $R$ of the primary. The dotted blue lines are explained in Figure 1 . The geometry of the eclipse is similar to that of $\zeta$ Aur but with a longer time-scale.

The bright K-supergiant $\zeta$ Aur binaries $31 \mathrm{Cyg}(\mathrm{K} 2 \mathrm{II}+\mathrm{B} 3 \mathrm{~V})$ and $32 \mathrm{Cyg}(\mathrm{K} 3 \mathrm{Ib}+\mathrm{B} 5 \mathrm{~V})$ also eclipsed in 2003, 32 Cyg again in 2006. Totality lasts 36 days in $\zeta$ Aur, and about 62 days in 31 Cyg. In 32 Cyg the eclipse is grazing, so totality only lasts about 5-7 days, depending on wavelength. The examples of extracted spectra of the secondary components of these binaries shown in Figures 2 and 3 illustrate particularly rapid changes in the profiles of the chromospheric Ca II K line. In all three systems the velocities of the chromospheric components are mostly well within the indicated limits of $\pm 80 \mathrm{~km} / \mathrm{s}$ that bracket the terminal wind velocities for the stars as reported in the literature.

All the spectra illustrated here, except those of $\zeta$ Aur in 1990, were observed with the DAO 1.2-m telescope and CCD camera at $2.4 \AA / \mathrm{mm}(R \sim 90,000)$.

\section{The Individuality of Stellar Chromospheres}

The three supergiant primaries are all of mid-K types, and stellar evolution theory treats them as rather similar objects. They have radii between $150-200 R_{\odot}$, and are approaching (if not on) the AGB. The secondary stars are all early-mid B dwarfs.

On the other hand, the regions sampled by each projected eclipse trajectory are somewhat complementary. The eclipse of $\zeta$ Aur occurs at a projected latitude on the giant 

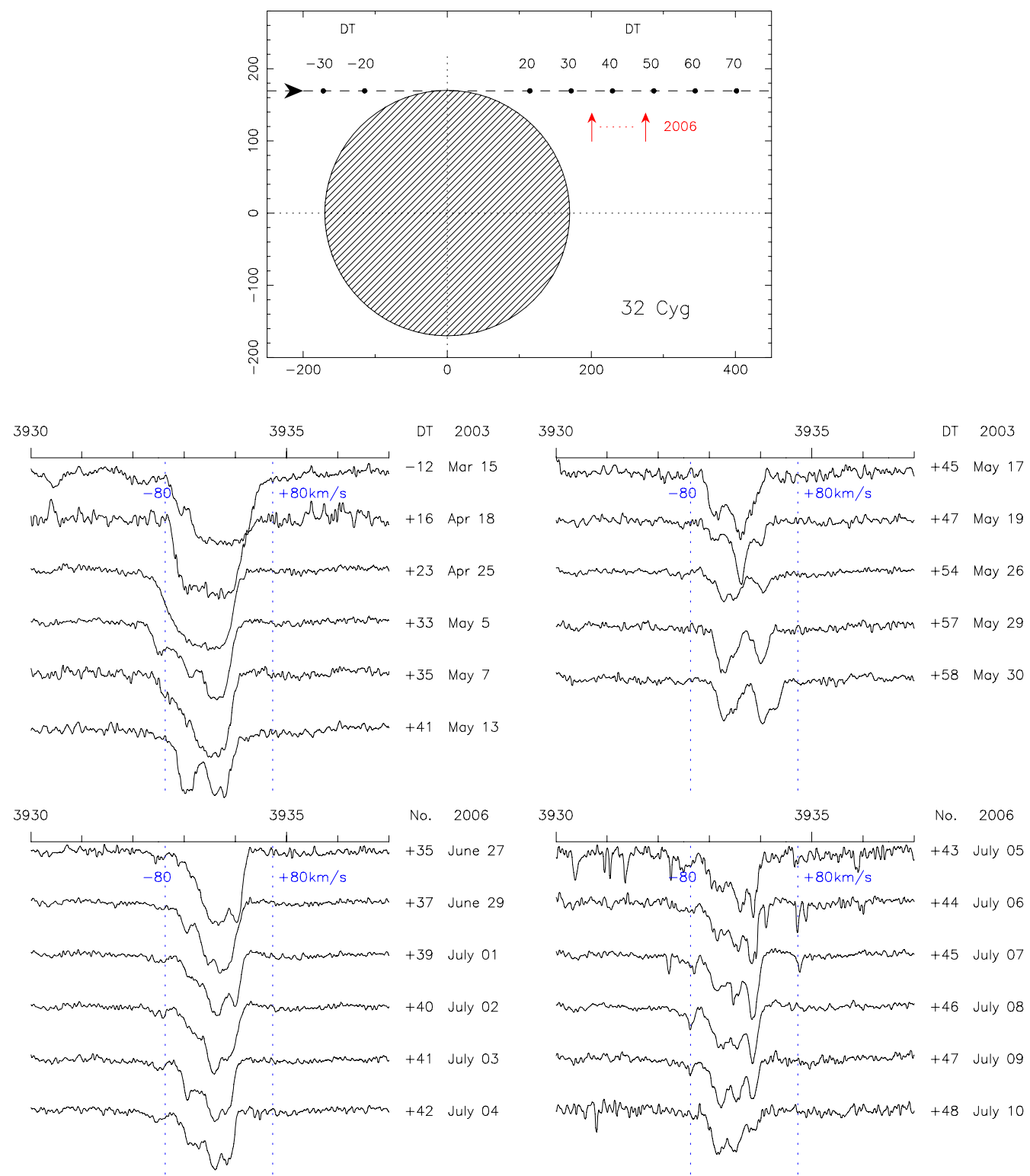

Figure 3. Top: Scaled eclipse model for $32 \mathrm{Cyg}$, in units of $R_{\odot}$, indicating the time-scale in $(D T)$ as defined in Fig. 1. Changes in the chromospheric K line in 32 Cyg at near-eclipse phases are shown for 2003 in the two middle spectra, and for 2006 in the lower pair. The red arrows in the model mark the range of $D T$ covered by the 2006 spectra. The dotted blue lines are explained in Figure 1.

of about $23^{\circ}$, so the light probe is sampling material along a path that is largely radial. The eclipse of $31 \mathrm{Cyg}$ is thought to be nearly central, whereas $32 \mathrm{Cyg}$ has a grazing eclipse and the light probe samples successively the "polar plumes" of the giant. Notice how much wider the chromospheric lines are in 32 Cyg compared to those in $\zeta$ Aur and 32 Cyg.

The relative transverse velocities between the respective giant and dwarf are not the 
same because the scales of the orbits are different. The secondary stars in both $\zeta$ Aur and 32 Cyg move through their own diameters in about 1.4 days, while in 31 Cyg it takes nearly twice as long. We should therefore not expect to see fresh chromospheric features appearing on adjacent days in any of the sequences. The fact that new ones do appear rapidly is an indicator of local velocities and activity.

\section{Activity in Supergiant Chromospheres}

The chromospheres of these three supergiants exhibit different scales of activity. Both 31 and 32 Cyg show considerably more random behaviour than has been detected so far in $\zeta$ Aur: the observed features suggest independent clouds, or polar streamers. Recall, too, the fineness of these details: as the models above demonstrate, each series corresponds to the projection of a rather narrow trajectory.

The double structure seen in $\zeta$ Aur during egress in 1990 and 2003 (but not in 1998) is curiously orderly, and its components are evenly divided in both strength and velocity. That rather precise symmetry, and its exact repetition on two occasions, is not in keeping with a model of independent clouds having random velocities. One possible interpretation is a superimposed emission such as a flare. However, since flares are random in both time and location the double structure could occur during ingress as well as egress, but so far it has only been seen during egress. More ingress and egress series are clearly needed. $\dagger$

In 31 Cyg there are dramatic changes with 24 hours - a much shorter interval than can be resolved by the light probe. Between 2003 April 3-6, for instance, it looks as though an independent cloud performed a "loop" around the more stable material, indicating that such clouds are not confined to meridional or radial movements but are also circulating transversely. However, there seemed to be much more activity during egress than ingress. For some 20 days (Aug 21 onwards) a strongly red-shifted cloud appeared and lingered, almost vanishing by Aug 28 but reappearing, stronger and doubled, a few days later. For about a week after that the various clouds are comparably dense and comparably separated in velocity, each about $25-30 \mathrm{~km} / \mathrm{s}$ apart. It will take at least another 21 years to establish whether the features seen in $31 \mathrm{Cyg}$ in 2003 are regular or not. I hope that some of the younger generation will become suitably enthused by the astrophysical potential of these observations!

The material in the polar regions of 32 Cyg appears to be considerably more turbulent than at the lower latitudes sampled in the other two supergiants; the broadening of the $\mathrm{K}$ line possibly masks its multi-component structure much of the time. Material occasionally appears at substantial velocities from the photosphere; in some spectra (not all included here) a cloud blue-shifted by about $85 \mathrm{~km} / \mathrm{s}$ appeared rather suddenly on 2003 May 5 and lingered for several days.

There is a huge opportunity here for studying the detailed behaviour of a star's outer atmosphere. The investment of the necessary dedicated telescope time on bright stars repays with spatially- and temporally-resolved details that are unique in stellar physics.

\section{Acknowledgements}

I am very grateful to the HIA/DAO and to the IAU for grants that have enabled me to attend the IAU GA, and to the DAO for numerous observing runs. I would also like to thank David Bohlender (DAO) for reducing the 2006 observations with his pipeline.

$\dagger$ Added in press: Spectra of the 2006 eclipse egress, observed during and after this Symposium, show some double structure in the $\mathrm{K}$ line but it is probably less symmetrical and less pronounced than in 2003. 